\title{
Patterns of hospital care for patients with HIV infection and AIDS
}

Academic Department of Genito-Urinary Medicine ${ }^{\star}$ and Department of Medicine, University College London Medical School, London W1N 8AA

A M Johnson

C Shergold

A Hawkins

$\mathrm{R}$ Miller

$M$ W Adler

Correspondence to: Dr A M Johnson * Academic Department of Genito-Urinary Medicine

Anne M Johnson, Carol Shergold, Anne Hawkins, Robert Miller, Michael W Adler

\begin{abstract}
Objective-The aims were to describe the use of inpatient resources by patients with HIV infection and patients with AIDS; to examine trends in service use over time; and to provide data for planners concerned with service provision for HIV infection.

Setting-An inner London health district reporting $9 \%$ of AIDS cases nationally. Design-Data on survival times and inpatient and day care use of resources were derived from existing patient records or collected prospectively between 1983 (when the first case of AIDS was diagnosed in the district) and 31 March 1990.

Subjects-A total of $488 \mathrm{HIV}$ positive patients of whom 396 had been diagnosed as having AIDS were studied.
\end{abstract}

Measurements and main results-Inpatient days consumed per annum; trends in the number of bed days per person year with AIDS; the lifetime inpatient use per AIDS patient; and the influence of survival on service use estimates were determined. Altogether $16.4 \%$ of a total 17785 hospital inpatient days were attributable to HIV positive patients who did not fulfil the criteria for AIDS. For patients with AIDS, there was an initial increase in the intensity of inpatient use in 1987 when a dedicated HIV ward was opened. After 1988, however, inpatient use fell to $30 \cdot 8$ bed days per person year with AIDS. Patients diagnosed after April 1987 had noticeably longer survival times than those diagnosed earlier (a median of 17-18 months compared with a median of 10-11 months). From 214 lifetime service use records, it was estimated that patients with short survival (less than six months) would consume 36 days of inpatient care, while those expected to survive longer would consume approximately twice that number of days, irrespective of how much longer they survived.

Conclusions-The data indicate less intensive use of inpatient care by AIDS patients over time, and hence the apparent ability to manage an increasing AIDS patient workload without a comparable increase in occupied bed days. Increases in the size of that workload and changes in the survival profile of patients, together with a relatively constant rate of service demand by longer survivors, however, will continue to place increasing strains on finite inpatient resources. Further research is needed to establish the extent to which the greater use of outpatient and community services can offset this.

f Epidemiol Community Health 1993; 47: 232-237

Studies of the impact of HIV infection on the health service have emphasised the costs of managing patients and the effect of the epidemic on hospital inpatient resources. ${ }^{1-3}$ Estimates of inpatient use $\mathrm{e}^{34}$ in this country have been based on small sample sizes and no recent studies have been published. Clinical reports indicate that the pattern of presentation and the length of survival of patients with AIDS have changed over the past few years in the UK, probably as a result of changes in clinical care such as earlier diagnosis, improved prophylaxis for and management of opportunistic infections, and the use of antiviral treatment. $^{5}$

At the same time there has been an increase in community care for patients with AIDS. This includes more involvement of general practitioners; the development of community care teams; greater use of hospital outpatient and day care as an alternative to inpatient care; and the development of hospice facilities for respite, palliative, and terminal care. ${ }^{6.8}$ There is little published evidence on the quantitative impact of this shift to community care on health service use or on its acceptability to consumers and providers. This information is important, not only for monitoring health service use but also for planning the provision of facilities for an increasing number of patients with HIV and AIDS. ${ }^{910}$

To provide estimates that could be used for monitoring and planning hospital services for patients with HIV infection, use of hospital inpatient resources had been studied in the Bloomsbury Health Authority (before its amalgamation with the Islington Health Authority). Bloomsbury Health Authority had reported $9 \cdot 1 \%$ of the recorded cases of AIDS in the UK up to the end of March 1990. Inpatient admissions for the management of clinical manifestations of HIV infection have been recorded since the first AIDS patient was diagnosed in the district in $1983 .^{3}$ Data from the period up to 1985 were used to plan a ward for patients with HIV. This ward was opened in April 1987. Since then a patient related database, backdated to 1983 , has been developed to include all patients with AIDS and all HIV related episodes in the district. Thus, information on temporal trends in workload and inpatient service use is available for examining evolving patterns of care. We report data from 1983 to March 1990, which comprises the records of 488 patients, all the patients with AIDS and all those 
admitted to hospital for care related to HIV infection.

\section{Methods}

Data Ease $^{11}$ software was used to establish a relational database to record details of cases of:

(i) AIDS treated in the district;

(ii) All patients requiring inpatient care for the management of HIV related illness, whether or not they fulfilled the criteria for the diagnosis of AIDS.

Centers for Disease Control definitions of AIDS were used throughout the study and the 1987 revision was adopted for all patients diagnosed after 1 September $1987 .{ }^{12}{ }^{13}$ To maintain confidentiality, no names were stored on the database and alpha-numeric codes were used as identifiers.

For all patients, data were collected on age, sex, area of residence, dates of diagnosis of HIV seropositivity and AIDS, dates of transfer in and out of the district, and date and place of death. Clinical data included the probable mode of HIV transmission and the AIDS indicator disease at diagnosis. Inpatient service use data included place of admission and dates of admission, discharge, and transfer. Data were compiled from clinicians' reports of AIDS cases, from ward returns, from the district patient administration system and from the genito-urinary medicine (GUM) outpatient system. At the time of an AIDS diagnosis, and at the time of death, patient administration system records and notes were checked to ensure that all inpatient episodes had been fully recorded, including those for HIV related disease before a diagnosis of AIDS had been made. Validation exercises were undertaken by crosschecking cases on the database against listings of AIDS case reports made to the CDSC, using alpha-numeric codes as identifiers. This analysis includes all patients with AIDS and all persons admitted for HIV related care from 1983 to 31 March 1990.

\section{INPATIENT EPISODES}

A patient was recorded as having an inpatien episode if he(she) occupied a bed at midnight for at least one night. Length of stay was calculated on the basis of the number of nights in hospital and included periods when the hospital bed was booked to that patient while he(she) was, for example, "on weekend leave".

A patient receiving day care on a hospital ward occupies a bed for a few hours only, if at all, and the episode has a length of stay of zero. Day care, has not therefore, been included in the total of hospital days used for HIV related care, or in the total number of discharges from hospital. Episodes of day care were not recorded consistently on the established hospital patient administration system before 1989. Data on day care before that date were collected from lists of patients supplied by clinicians and from clinical notes.

SERVICE USE TIME AND SURVIVAL

For inpatient stays which began before 31 March 1990 but terminated after that date, only those inpatient days that fell within the specified study period were included in service use estimates.
Where the specific date of diagnosis, transfer in, transfer out, or death, were not available, the event date was recorded as the 15 th of the relevant month, or more precisely where possible. Patients who began treatment in Bloomsbury after AIDS had been diagnosed were treated as tranfer-in patients. Patients were classified as transfer-out patients if they were known to have transferred their care to another district or country. The date on which each patient was last seen in one of the district's services was recorded and provided the basis for truncating the survival times of those patients for whom no date of death or date of transfer out to another district was known. Patients who were lost to follow up were included in the analyses using a "last known alive" date.

For patients first diagnosed as fulfilling the criteria for AIDS during an inpatient episode, the date of diagnosis of AIDS was taken to be the day of admission for that stay. All inpatient days that occurred after the date of the AIDS diagnosis were considered to be AIDS related. The remaining days recorded were for patients with a diagnosis of HIV seropositivity who did not fulfil the criteria for AIDS.

During the study period five patients who self reported HIV seropositivity were admitted to hospital. These patients were subsequently found to be seronegative and have been excluded from analyses.

Overall trends in HIV and AIDS related stays were examined from the first case of AIDS in 1983 to 31 March 1990. Service use for patients fulfilling an AIDS diagnosis was examined in greater detail. Trends in inpatient use were examined by relating the AIDS workload (total number of AIDS patients being treated) by quarter to indices of service use. These include the number of inpatient days, number of inpatient episodes, and lengths of stay per episode. The total number of inpatient bed days, and day care episodes used per person year (or per person quarter) with AIDS for each financial year from $1982 / 3$ to 31 March 1990, were also examined.

Changes in survival of patients with AIDS were examined using Kaplan-Meier survival analysis techniques. ${ }^{14}$ The influence of both personal survival time and also date of diagnosis were examined in relation to service use. Lifetime service use was examined in a subgroup of 214 AIDS patients who received all their care in Bloomsbury and for whom completed records of lifetime service use were available.

\section{Results}

Altogether 488 individuals were identified who had received an AIDS diagnosis, inpatient treatment, or day care in hospital for HIV infection between 1983 and 31 March 1990. Four fifths of these patients were homosexual men. Only 15 were women, eight of whom were diagnosed as having AIDS. By the end of March 1990, eight patients had died without fulfilling the criteria for an AIDS diagnosis. In all, 396 patients were diagnosed with AIDS before the end of the study period. The initial AIDS diagnosis was Pneumocystis carinii pneumonia in $169(43 \%)$ of patients; Kaposi'a sarcoma in 94 (24\%); other opportunistic infections in 42 (11\%); HIV 
dementia in eight $(2 \%)$; and other tumours in 13 (3\%). A further 38 patients $(10 \%)$ had two or more AIDS indicator diseases present at diagnosis. Information on initial diagnosis was missing for the remaining 32 patients $(8 \%)$.

Of the total of 17785 hospital inpatient days recorded, $82.6 \%$ were for patients with an AIDS diagnosis at the time of their inpatient episode. The proportion of hospital days used by HIV positive patients without an AIDS diagnosis rose,

Table I Inpatient days for HIV positive patients who did not fulfil an AIDS diagnosis as a percentage of all days for HIV related care

\begin{tabular}{lccc}
\hline & \multirow{2}{*}{$\begin{array}{c}\text { All stayst } \\
\text { (Bed days) }\end{array}$} & \multicolumn{2}{c}{ HIV positive (non-AIDS) stays } \\
\cline { 3 - 4 } \cline { 3 - 4 } Beriod days & B of all stays \\
\hline $1983-5$ & 794 & 61 & $7 \cdot 7$ \\
$1985-6$ & 1032 & 68 & $6 \cdot 6$ \\
$1986-7$ & 2000 & 341 & $17 \cdot 0$ \\
$1987-8$ & 4928 & 641 & $13 \cdot 0$ \\
$1988-9$ & 4251 & 890 & $20 \cdot 9$ \\
$1989-90$ & 4780 & 916 & $19 \cdot 2$ \\
Total & 17785 & 2917 & $16 \cdot 4$ \\
\hline
\end{tabular}

*All periods ending 31 March.

†Diagnostic data missing on $176(1 \%)$ of bed days.

A

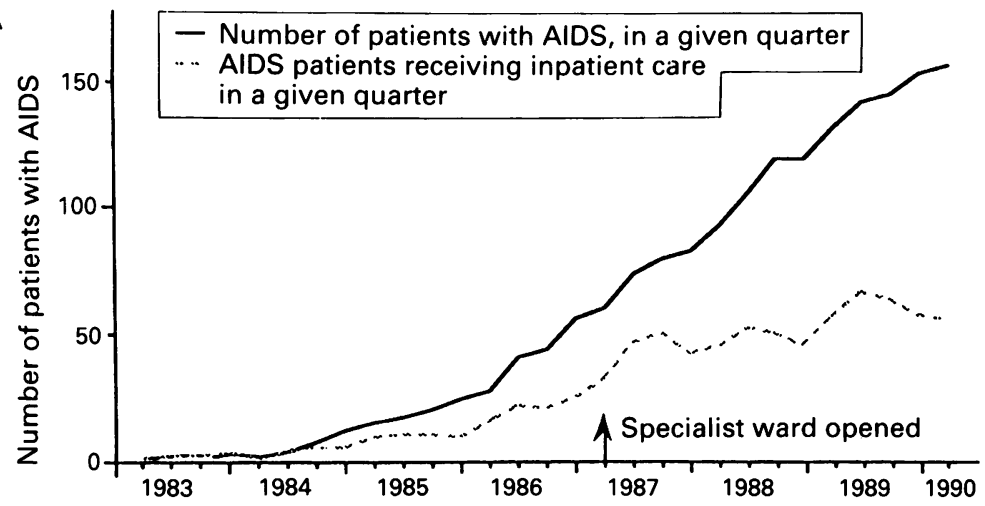

B

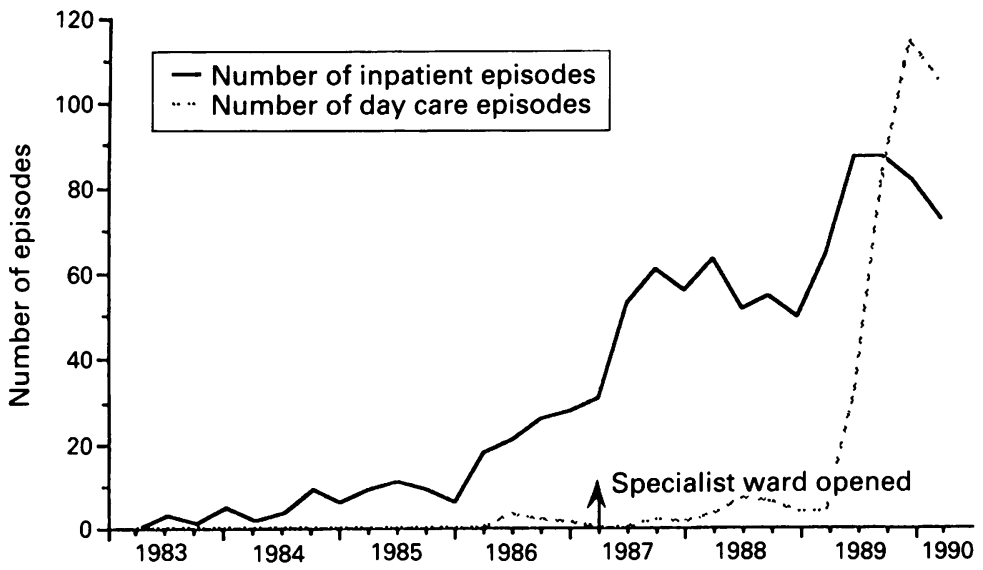

C

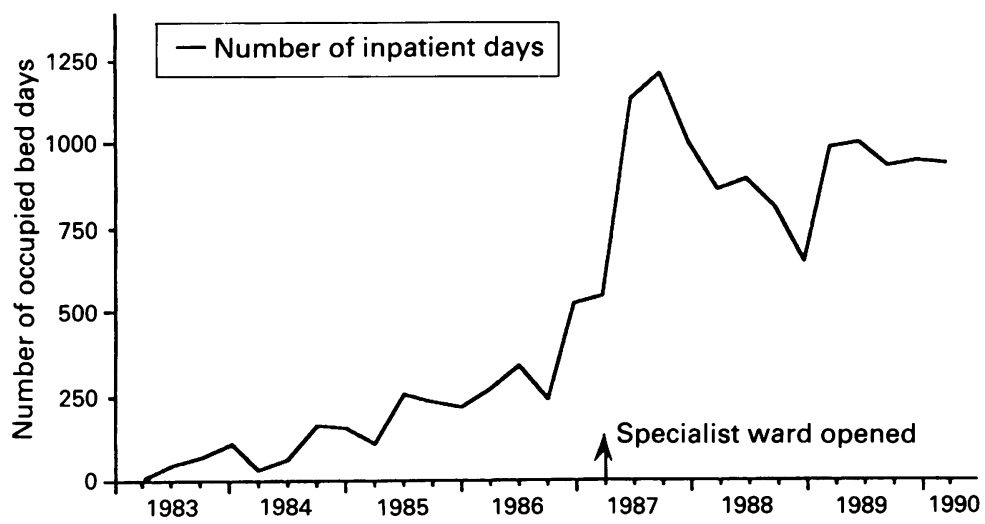

Figure 1 Trends in AIDS case workload by quarter year from 1983 to 31 March 1990. however, from less than $10 \%$ before April 1986 to over $19 \%$ in the financial year 1989-90 (table I).

Although $280(71 \%)$ of the 396 patients diagnosed with AIDS had their first episode of inpatient care at the time of, or after, a diagnosis of AIDS had been made, $60(15 \%)$ received inpatient care both before and after their AIDS diagnosis. A further $13(3 \%)$ had so far received inpatient care only before their AIDS diagnosis; eight $(2 \%)$ had received day care only; and only 35 (9\%) had had no inpatient admissions or day care thus far. Of the $92 \mathrm{HIV}$ positive patients not fulfilling an AIDS diagnosis at 31 March 1990, 84 had received inpatient care and eight had received day care only.

Clearly then, patients who did not yet fulfil a diagnosis of AIDS counted for a substantial minority of bed days devoted to inpatient care. They represent a poorly defined population of HIV positive patients treated in the district but not enumerated. Further analysis of trends in care was therefore confined to cases of AIDS, for which both the number of cases and the periods of treatment could be clearly defined.

Figure 1 shows the AIDS case workload (total number of AIDS patients being treated by quarter, shown in figure $1(\mathrm{~A})$ ), together with quarterly trends in the following indices of service use:

Number of AIDS patients receiving inpatient care (figure $1(\mathrm{~A})$ );

Number of inpatient episodes (figure 1(B));

Number of day care episodes (figure 1(B));

Number of occupied bed days (figure 1(C)).

The AIDS case workload rose from one for the quarter ending 31 March 1983 to 157 for the quarter ending 31 March 1990. Over the same period, the number of episodes of inpatient care rose from 0 to 73 , having been above 80 for the preceding three quarters (figure $1(\mathrm{~B})$ ). Day care service began to affect a noticeable number of patients only from April 1989 onwards but rose to a maximum of 115 episodes in the last quarter of 1989 (figure 1(B)).

The number of inpatient bed days used per quarter rose from seven in the second quarter of 1983 to 942 in the first quarter of 1990 (figure $1(C)$ ). Before 1987 , the rise was relatively gradual. As in each of the graphs shown in figure 1, however, it is possible to discern some measure of discontinuity occurring at the time that the dedicated HIV ward was opened in 1987. After that, the number of inpatient bed days used seems to have stabilised to a fairly consistent level for the last year of the study period. It is evident that although the AIDS case workload went on rising steadily, more than doubling from 61 in the quarter ending 31 March 1987 to 157 patients in the quarter ending 31 March 1990, there was no equivalent increase in the number of bed days used.

Figure 2 shows that the average number of inpatient days per AIDS patient quarter fell from more than 20 in 1983 to six in the first quarter of 1990. During the same period, the average length of stay per episode fell more gradually, reaching a level of 11-12 days by the end of the study period.

As the average number of inpatient episodes per person year with AIDS also fell over the same period (from 4.9 before 1985 to 2.7 in $1989 / 90$ ) 


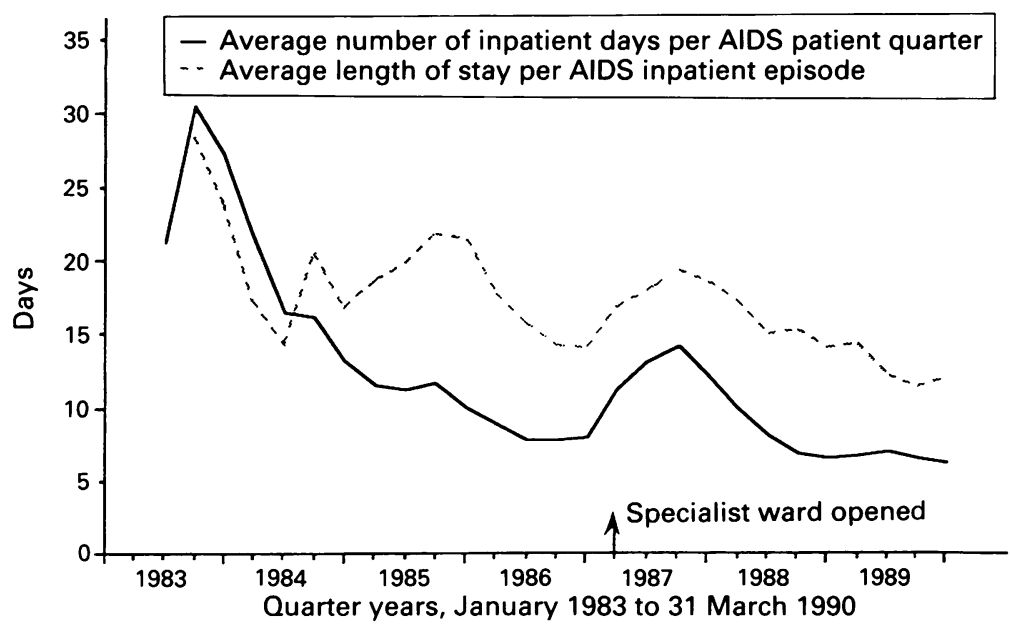

Figure 2 Average number of inpatient days per AIDS patient quarter and average length of stay per AIDS inpatient episode, for quarter years 1983 to 31 March 1990, smoothed by a factor of 3 .

the apparent increase in efficiency of bed use may be accounted for by a combination of falling length of stay and decreased frequency of admission (table II). The number of inpatient days used per patient year with AIDS was over 90 before 1985 but had fallen to $30 \cdot 8$ days per person year with AIDS in the year 1989-90. There is again discernible discontinuity in this trend, however, after the opening of the ward in 1987.

Service use may be influenced by medical practice, by patient characteristics, and by survival time. Any or all of these may themselves be dependent on the time that has elapsed since the start of the epidemic, thereby complicating the investigation of time based changes in service use. A Kaplan-Meier survival analysis for cohorts of patients diagnosed in different years showed significant differences in survival times (log rank $\mathrm{p}=0.0003$, Wilcoxon $\mathrm{p}=0.0001$ ) but no linear trend. It seemed that patients diagnosed after April 1987 had appreciably longer survival times

Table II Inpatient (IP) and day care days per person year (PPY) with AIDS

\begin{tabular}{lcclll}
\hline Period $^{*}$ & $\begin{array}{l}\text { Person years } \\
\text { with } \text { AIDS }\end{array}$ & IP days & $\begin{array}{l}\text { IP days } \\
\text { PPY }\end{array}$ & $\begin{array}{l}\text { IP episodes } \\
\text { PPY }\end{array}$ & $\begin{array}{l}\text { Day care } \\
\text { days } P P Y\end{array}$ \\
\hline Before 1985 & $7 \cdot 99$ & 733 & $91 \cdot 72$ & $4 \cdot 88$ & 0 \\
$1985-6$ & $17 \cdot 97$ & 964 & $53 \cdot 64$ & $2 \cdot 45$ & 0 \\
$1986-7$ & $38 \cdot 74$ & 1646 & $42 \cdot 49$ & $2 \cdot 74$ & $0 \cdot 15$ \\
$1987-8$ & $66 \cdot 48$ & 4199 & $63 \cdot 16$ & 3.52 & 0.09 \\
$1988-9$ & $99 \cdot 41$ & 3326 & $33 \cdot 46$ & $2 \cdot 23$ & 0.21 \\
$1989-90$ & $124 \cdot 24$ & 3824 & $30 \cdot 78$ & $2 \cdot 66$ & $2 \cdot 70$ \\
\hline
\end{tabular}

*All periods ending $31 \mathrm{March}$

Table III Average proportions of AIDS survival time spent receiving inpatient care by survival time and year of diagnosis * (number of patients) and expected lifetime inpatient carc

\begin{tabular}{|c|c|c|c|c|c|}
\hline \multirow[b]{2}{*}{ Diagnosis datet } & \multicolumn{5}{|c|}{ Patients' survival times $(\mathrm{m})$} \\
\hline & $<6$ & $6-12$ & $12-18$ & $18-24$ & $>24$ \\
\hline $\begin{array}{l}1983-5 \\
1985-6 \\
1986-7 \\
1987-8 \\
1988-9 \\
1989-90\end{array}$ & $\begin{array}{l}0.60(10) \\
0.76(1) \\
0.32(22) \\
0.54(16) \\
0.37(20) \\
0.39(15)\end{array}$ & $\begin{array}{l}0.27(6) \\
0 \cdot 16(4) \\
0 \cdot 22(10) \\
0.35(11) \\
0.20(10) \\
0.19(5)\end{array}$ & $\begin{array}{l}0 \cdot 12(3) \\
0 \cdot 15(9) \\
0 \cdot 15(8) \\
0 \cdot 20(7) \\
0 \cdot 12(10) \\
-\end{array}$ & $\begin{array}{l}0.01(1) \\
0.15(3) \\
0.12(5) \\
0.11(15) \\
0.13(3) \\
-\end{array}$ & $\begin{array}{l}0.06(2) \\
0.09(3) \\
0.06(11) \\
0.07(4) \\
- \\
-\end{array}$ \\
\hline Overall & $0.43(84)$ & $0 \cdot 25(46)$ & $0 \cdot 15(37)$ & $0 \cdot 11(27)$ & $0.07(20)$ \\
\hline $\begin{array}{l}\text { Average } \\
\text { survival (d) }\end{array}$ & $83 \cdot 2$ & $275 \cdot 6$ & $456 \cdot 4$ & 617.0 & $984 \cdot 9$ \\
\hline $\begin{array}{l}\text { Expected lifetime } \\
\text { inpatient care }(\mathrm{d}) \ddagger\end{array}$ & 36 & 69 & 68 & 68 & 69 \\
\hline
\end{tabular}

than those diagnosed earlier (median 17-18 months compared with 10-11 months). This may reflect possible earlier diagnosis and improved treatment. A similar analysis of survival times for patients grouped according to the initial AIDS diagnosis also showed significant differences (log rank and Wilcoxon $\mathrm{p}=0 \cdot 0001)$. There was no significant association between the year of diagnosis and the type of indicator disease.

Table III shows the influence of survival time on the intensity of service use for the 214 AIDS patients for whom completed service use records were available. Those patients who had shorter survival times spent a higher proportion of time in hospital, but from diagnosis to death used significantly less inpatient care $\left(\mathrm{F}_{4,188}=13 \cdot 6, \mathrm{p}<0 \cdot 001\right)$. In particular, a patient who survived for less than six months could be expected to consume in the order of 36 inpatient days from diagnosis to death, while a patient who survived for longer than six months could be expected to consume nearly twice that number of days, irrespective of how much longer their survival was.

The overall average number of inpatient bed days used from diagnosis to death was 51.6 days. Such an overall statistic must be treated with caution, however, because of the considerable variability shown in table III associated with both survival time and diagnosis year. Also, the use of completed service use records tends to bias the outcome towards short term survivors.

As the epidemic has progressed, there have been concomitant changes in survival profiles of the AIDS patients making up the case workload. Initially, the AIDS caseload was comprised of larger proportions of patients who, up to that time, had had shorter survival times. By the end of the study period, however, the caseload had become more evenly distributed with regard to the survival times of the AIDS patients then receiving care, such that there was a greater proportion of patients in care with longer survival times. In view of the differences in service use for short and longer surviving patients, estimates of inpatient service needs for the future must take account of the likely survival profile of the caseload. Furthermore, changes in this distribution of survival times will influence the intensity of service use by influencing the proportions of patients admitted to terminal inpatient care. The mean length of inpatient episodes ending in death (19 days) was significantly longer $\left(F_{1,699}=5.45, p=0.02\right)$ than those ending in discharge (15.1 days).

\section{Discussion}

This study has documented the use of inpatient resources by patients with AIDS and HIV infection over a seven year period, and provides summary data that may be used for planning resource use for the increasing number of patients with AIDS in the UK. We have emphasised that patterns of inpatient service use have not been stable over time and have attempted to analyse some of the components and determinants of change.

The data support the view that at least since 1987, the overall intensity of service use by patients with AIDS has diminished. There has been a falling rate of use of inpatient days per 
AIDS patient year. The financial year 1987-8, however, was perhaps atypical, with the opening of a purpose built ward for patients with HIV and AIDS. In that year the use of inpatient days per person year with AIDS increased before falling rapidly in the following years. The apparent reduction in the intensity of service use has permitted the district to manage a considerably increased workload of AIDS patients without increasing its bed capacity over the course of three years. In 1990, however, a further 15 bed ward was opened for both patients with HIV and chest diseases, and we will continue to monitor service use now that this further capacity is available.

The decreased intensity of service use reflected both a falling length of stay per episode and also a reduced frequency of inpatient admission (figure 2 , table III). Several factors might account for these changes. These include changes in availability of beds (such as occurred when a dedicated ward was opened); changes in treatment regimens (including physician experience, prophylactic therapy, and possible earlier diagnosis); the development of a local community care team and terminal care facilities outside of the hospital ${ }^{7}$; and the influence of changing survival profiles of successive cohorts of AIDS patients. The intensity of service use was influenced considerably by survival time (table III) such that those with short survival times spent a much higher proportion of their time with AIDS in inpatient care than did those with longer survival, but overall consumed fewer hospital days than longer survivors. From the 214 lifetime service use records, our estimated lifetime service use was 36 days for those surviving less than six months, but rose to 68 or 69 days, and remained remarkably stable, for those surviving longer than six months. Despite the reduced intensity of inpatient use for longer survivors, the proportion of these patients within the AIDS workload has increased. Consequently, there is now an increased proportion of patients who could be expected to require terminal (and therefore longer) inpatient care, a need which may partly be met by the introduction of terminal care facilities outside hospital. These changes, combined with the increasing numbers of patients with AIDS, emphasise that the overall burden on the health service can be expected to continue increasing. ${ }^{9}$

For AIDS patients with complete service use records, the overall average number of inpatient bed days used from diagnosis to death was 51.6 days. Although overall statistics may yield a general impression of expected service use, they should be treated with caution because of the considerable variability in use which was observed over the time period of this study and the potential bias towards short term survivors. This is particularly noticeable for those diagnosed most recently and is a problem inherent in methods that consider only completed service records. ${ }^{15}$ The lifetime figure is similar to that recorded in an earlier study from the district of 50 days, in the first 33 patients diagnosed in the district. ${ }^{3}$

In common with other observers, we attempted to overcome the difficulties inherent in lifetime service use estimates by also examining service use per person year with AIDS for the full group of 396 AIDS patients. ${ }^{2}$ We have shown a reduction in inpatient care per AIDS patient year to 30.8 days in 1989-90. This lies at the lower end of range estimates of 19-155 days from earlier surveys in the USA and Europe. ${ }^{2}$ More recently, data from a 1987 cross sectional hospital survey in the USA estimated that the average patient with AIDS received 26 days of inpatient care per patient per year. ${ }^{1}$ These figures cannot be compared directly with those in the present study because they are not based on analysis of service use per person year and take no account of variability in survival. In an analysis similar to our own, in patients diagnosed in California, Queensberry et al ${ }^{15}$ reported consumption of 31 inpatient days per AIDS patient year in 1987. They reported very similar results to ours showing decreased intensity of service use with increasing survival, but little effect of year of diagnosis on lifetime use.

As the intensity of inpatient use declines and survival increases, it is evident that patients with AIDS will spend an increasing amount of time out of hospital. While many may be relatively well, continuing in employment and maintaining independent lives, they will require increased outpatient resources for monitoring of disease progression; prophylactic and therapeutic intervention; and counselling. Those who are sicker may require increased primary care and community support, shifting care to community services, family, and informal networks.

While our analysis has focused on care of AIDS patients, it should be emphasised that in 1989-90 nearly one fifth of the bed days devoted to care of patients with HIV were for those who did not fulfil an AIDS diagnosis. Not only is it necessary to plan for this extra care, but in allocating district resources for the care of people with HIV infection, account should also be taken of this usage. Current allocations do not tend to consider this as they are based largely on the number of AIDS cases reported.

The available data on day care indictate that this is becoming an increasingly popular option, reflecting clinical use for investigative procedures, blood transfusion, and nebulised pentamidine for pneumonia prophylaxis. These options as an alternative to inpatient care are not necessarily cheaper, however, and day care must therefore be included in resources estimates.

Data on outpatient service use was not collected routinely until 1988. In our district most outpatient care for HIV positive patients is undertaken within the GUM clinic which now collects data on outpatient visits for patients with HIV and AIDS for statutory returns (KC60) to the Department of Health. These indicate that in 1989-90, in addition to inpatient care, those who were HIV positive and asymptomatic required an average of 3.5 episodes of outpatient care and those with symptomatic HIV infection and AIDS required 5.7 episodes of outpatient care in the GUM clinic. These accounted for a total of over 4500 outpatient visits during the year.

Despite a reduction in the intensity of inpatient resource use, patients with AIDS and HIV are still frequent users of hospital resources. Continued monitoring will enable us to provide continuing data for service planning. We have not, however, examined outcomes other than service use, and 
our data describe the actual, and not necessarily the optimal, consumption of resources. Considerable financial investment and innovative service development has occurred in response to AIDS. Further evaluation of different models of service use taking into account issues such as patient satisfaction, quality of life, and outcome of care, as well as resource consumption, would make a valuable contribution to health services research in this field.

This work was supported by a grant from the Department of Health. Anne Hawkins is supported through the MRC Co-ordinating Centre for Epidemiological Studies of AIDS and HIV. We also thank Cathy Walter and Chris Bentley for their assistance and Kay Stratton for secretarial support.

1 Andrulis DP, Beers Weslowski V, Gage LS. The 1987 US

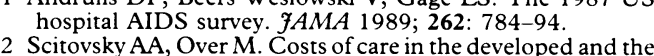
Scitovsky AA, Over M. Costs of care in the developed a
developing world. AIDS 1988; 2 (suppl 1): S71-81.

3 Johnson AM, Adler MW, Crown JM. AIDS and the Johnson AM, Adler MW, Crown JM. AIDS and the
epidemic of infection with HIV: costs and prevention in an epidemic of infection with HIV: costs and preven
inner London district. BMF 1986; 293: 489-92.
4 Cunningham D, Griffiths SF. AIDS: counting the cost. BMIf 1987; 295: 921-2.

5 Peters BS, Beck EJ, Coleman DG et al. Changing disease patterns in patients with AIDS in a national centre in the United Kingdom: the changing face of AIDS. BMIf 1991; 302: 203-7.

6 Bould M, Peecock G, eds. AIDS: models of care. London: King's Fund Centre, 1989.

7 Butters E, Higginson I, George R, Smits A, Wede A, Butters E, Higginson I, George R, Smits A, Wede A,
McCarthy M. Two community HIV/AIDS teams: referrals, patients characteristics and pattern of care. Health Trends (in press).

8 Smits A, Mansfield S, Singh S. Facilitating care of patients with HIV infection by hospital and primary care teams. $B M I F$ 1990; 300: 241-3

9 Report of a working group convened by the Director of Public Health Laboratory Service. Chairman: Professor NE Day. AIDS in England and Wales to end 1993. Projections using data to end September 1987. CDR, 1990, 1-12.

10 Bentley C, Adler MW. Choice cuts for patients with AIDS. BMF 1990; 301: 825-6.

11 Data Ease 4.0 rl. 1989. Dataease International Inc, USA.

12 Centers for Disease Control. Revision of case definition of acquired immunodeficiency syndrome for national reporting-United States. MMWR 1985; 34: 373-5.

13 Centers for Disease Control. Revision of the CDC surveillance case definition for acquired immunodeficiency survellance case definition for acquired
syndrome. $M M W R$ 1987; 36: 3S-15S.

syndrome. $M M W R$ Additional $S A S / S T A T$ procedures, release 6.03. Cary: SAS
Institure Institute Inc, 1988. Technical report P-179.

15 Queensbury CP, Fineman B, Hiatt RA, Selby JV. A survival Queensbury CP, Fineman B, Hiatt RA, Selby $\mathrm{N}$. A survival
analysis of hospitalisation among patients with acquired immunodeficiency syndrome. Am f Public Health 1989; 79: $643-7$ 